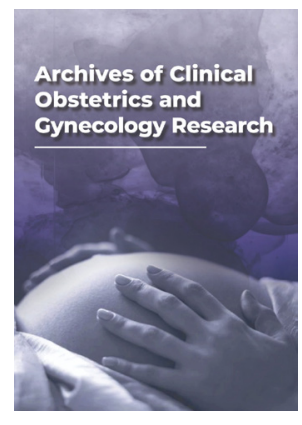

Correspondence

Linda L Remy

UCSF Family Health Outcomes Project, 500 Parnassus Ave. Room MU-337, San Francisco, California 94143-0900, USA

Tel.: +1 415-476-5283

Fax: +1 415-476-6051

E-mail: Linda.Remy@ucsf.edu

Website: https://fhop.ucsf.edu

- Received Date: 25 Aug 2020;

- Accepted Date: 03 Sep 2020;

- Publication Date: 08 Sep 2020.

\section{Keywords}

Gestational diabetes mellitus; Diabetes mellitus in pregnancy; Adverse pregnancy outcomes; Longitudinal trends

\section{Copyright}

(C) 2020 Science Excel. This is an openaccess article distributed under the terms of the Creative Commons Attribution 4.0 International license.

\title{
Longitudinal trends for diabetes during pregnancy: California 1983-2015
}

\author{
Linda L Remy and Ted Clay \\ UCSF Family Health Outcomes Project, San Francisco, California, USA
}

\begin{abstract}
Objective: To report longitudinal trends (1983-2015) for pregnant women hospitalized in California with pre-existing Type 1 or Type 2 diabetes mellitus (PDM) and gestational diabetes mellitus (GDM). Research design and methods: Using 1983-2015 patient discharge data, we identified admissions of pregnant California resident women, age 15 to $44(\mathrm{~N}=18,560,269)$. We used the Clinical Classification System to classify diagnoses and procedures.

Results: Over the period, 18,952,079 pregnant California-resident women age 15 to 44 were admitted to hospital. Of these, 212,631 (1.1\%) had PDM and 787,361 (4.2\%) had GDM. At start-of-period 19831987 , the percent of admissions with either condition was about 1\% each. By 2013-2015, the PDM rate rose to $1.5 \%$ while the GDM rate rose to $9.1 \%$. Compared to women without diabetes, those with any diabetes had greater risk and rising trends for co-morbidities and adverse outcomes.

Discussion: It is not clear if these trends reflect real underlying changes in population health or changes in professional attention to these conditions. Trends may be rising because of different diagnostic criteria, because of underlying changes in risk factors, or for both reasons.

Conclusions: Regardless of the underlying reason, PDM and GDM pose significant risk to a growing proportion of California's pregnant women.
\end{abstract}

\section{Introduction}

Diabetes mellitus is a group of metabolic diseases characterized by hyperglycemia resulting from defects in insulin secretion, insulin action, or both [1]. The United States Centers for Disease Control and Prevention estimates that $4.1 \%$ of the population age 20 to 44 has diabetes mellitus, with women slightly less likely to have it [2]. This condition affects many parts of the body and is associated with serious complications such as heart disease, stroke, blindness, kidney failure, and lower-limb amputation.

Gestational diabetes mellitus (GDM), defined as any degree of glucose intolerance with onset or first recognition during pregnancy [3], represents nearly $90 \%$ of pregnancies complicated by diabetes mellitus [1], and today complicates about $7.6 \%$ of US pregnancies $[4,5]$. GDM affects both maternal and infant outcomes [6]. Women with GDM in one pregnancy are at increased risk in later pregnancies $[7,8]$.

Both pre-existing Type 1 or Type 2 diabetes mellitus (PDM) and GDM are associated with significant risk of poor outcomes in the current pregnancy and future risk of obesity and diabetes in the offspring. There is a strong association between maternal diabetes and impaired cognitive outcomes for the infant such as learning disabilities and autism $[9,10]$.

In 1979, the National Diabetes Data Group (NDDG) developed and published the classification and diagnosis of diabetes used in the US [3]. Although members defined GDM and proposed new diagnostic criteria, the NDDG felt that the diagnostic criteria had not been widely tested and therefore made no new recommendations. In 1980, the International Classification of Disease (ICD) changed from Version 8, which did not distinguish GDM, to Version 9, which for the first time introduced specific GDM codes. As Lavery and colleagues noted, screening and diagnostic criteria evolved over the past several decades with five international workshops since 1979 [11], and these changes complicate interpretation of longitudinal studies.

This brief compares PDM and GDM trends for pregnancy admissions for the period 1983 through Sep-2015, during which California used the ICD-9$\mathrm{CM}$ to report patient diagnose in hospital discharge data. The conversion to ICD-10-CM in Oct-2015 provided an opportunity to do this longitudinal report.

\section{Methods}

Using previously described methods [12,13] and the Jan-1983 to Sep-2015 confidential patient discharge data from the California Office of Statewide Health Planning and Development (OSHPD) [14], we identified all inpatient admissions of pregnant California resident women, age 15 to $44(\mathrm{~N}=18,560,269)$, including those that did not result in live birth.

To classify diagnoses (DX) and procedures (PX), we applied the Clinical Classification System (CCS) developed by the Agency for Healthcare Research and Quality [15]. CCS Level 2 groups diagnoses (DXCL) and procedures (PXCL) within 
body systems. Searching over the array of diagnoses, PDM was defined as DXCL codes 49 or 50 or DX 648.0x under DXCL186. GDM was defined as cases under DXCL186 with no PDM [16].

In the period 1983-2007, the selection algorithm flagged pregnancy as the primary reason for admission if the Diagnosis Related Group (DRG) was in the range of 370-384. The Medicare Severity DRG (MSDRG) took effect in 2008, after which the algorithm flagged pregnancy if the MSDRG was in the range 765 to 782 . However, a number of women need hospital care for reasons not related directly to pregnancy. For example, admission with a principal diagnosis of injury, mental health, or infectious disease raises pregnancy risk. Identifying these cases involves searching the array of DX and PX fields. Consider a woman admitted with MSDRG indicating injury or mental health as the primary reason for admission. Searching the DX array identifies that she also was pregnant (DXCH11) and that the pregnancy ended with a spontaneous abortion (DXCL177). Relying on DRG or principal DX to identify pregnancy status would not find such records.
The analysis was completed in SAS, version 9.4. Supplemental File 1 reports frequencies, overall percent distributions, with odds ratios and confidence intervals comparing differences between admissions with PDM or GDM to admissions without these conditions, and differences between PDM and GDM. These were calculated using SAS PROC FREQ with the CMH option. To highlight both clinical and statistical significance, odds ratios are bolded when the P-value is greater than 0.001 and the confidence interval is plus/minus 0.05 [17]. The Supplemental File also contains data used to prepare figures.

\section{Results}

Over the 33-year interval 1983 through Sep-2015, the selection algorithm identified 18,952,079 admissions of pregnant Californiaresident women age 15 to 44 . Of these, 212,631 (1.1\%) had PDM and 787,361 (4.2\%) had GDM. The table in Supplemental File 1 reports overall demographic, access, and outcome characteristics. We focus here on longitudinal trends for selected measures.

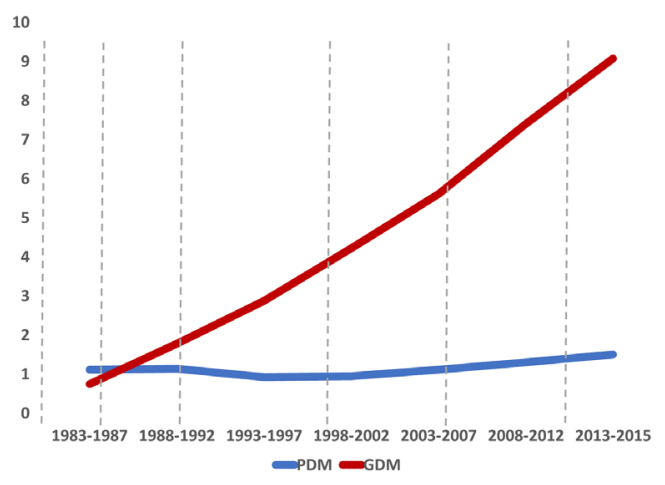

Figure 1. Admissions with PDM or GDM (\%)
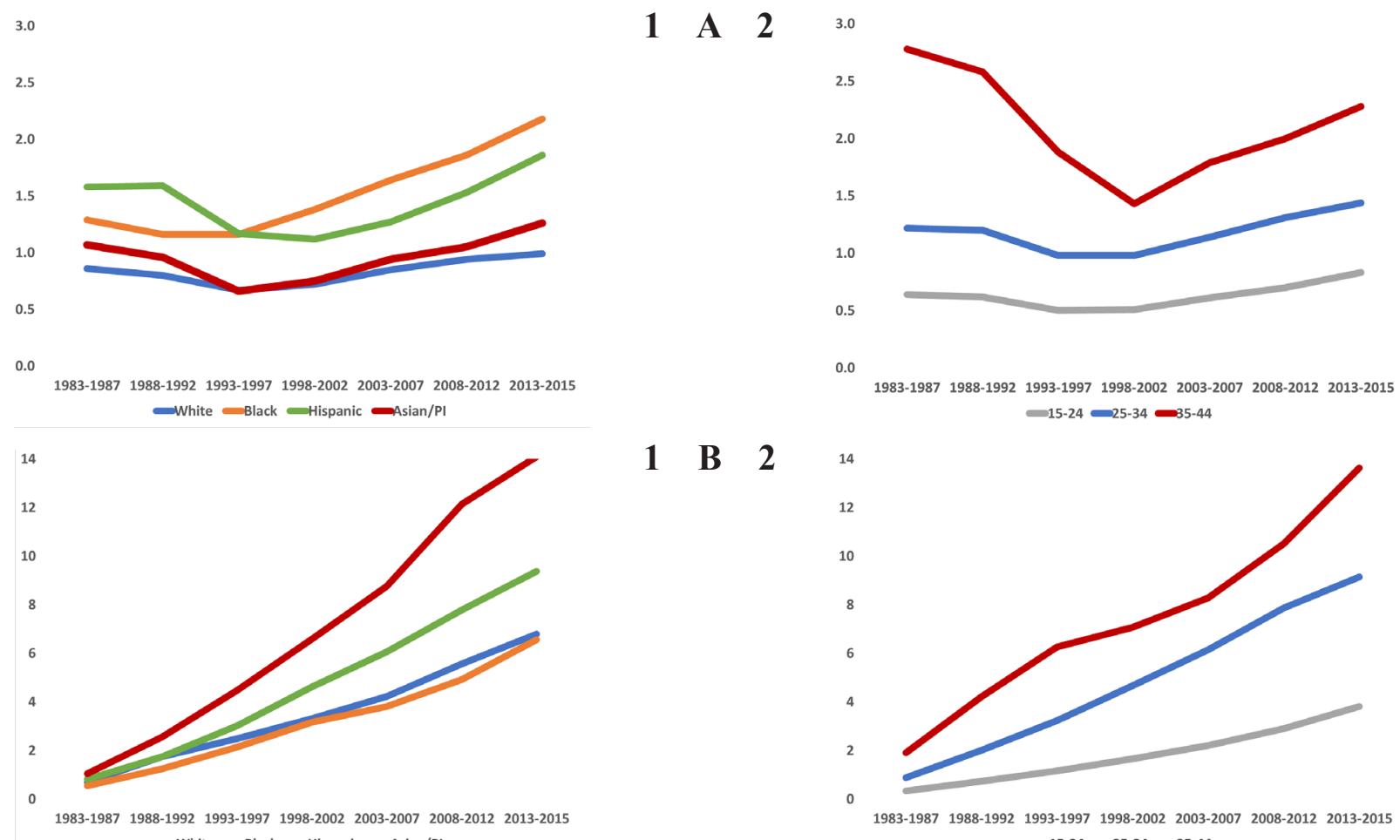

1 B 2

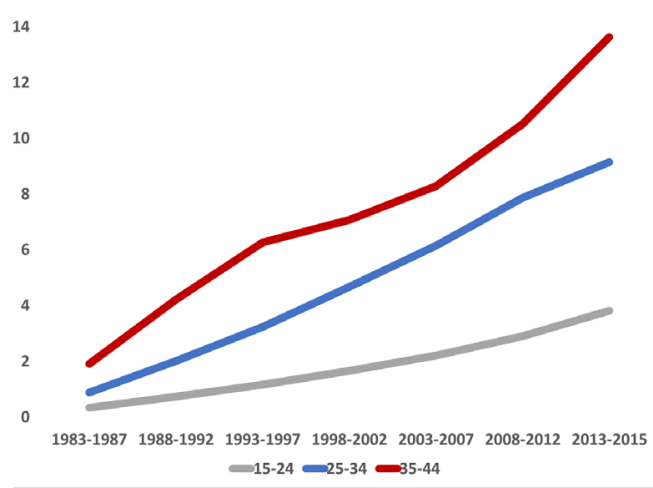

Figure 2. Demographic trends per 100 pregnancy admissions (\%) 1983-2015

A. Pre-existing diabetes mellitus (PDM) [1. Race/Ethnicity; 2. Age group]

B. Gestational diabete;s mellitus (GDM) [1. Race/Ethnicity; 2. Age group] 
Figure 1 shows the trend for the percent of pregnant hospitalized women diagnosed with PDM or GDM over the study period. At the start-of-period 1983-1987, the percent of admissions with either condition was about $1 \%$ each. The PDM rate rose to $1.5 \%$. However, by $2013-2015$, the GDM rate rose to $9.1 \%$. Vertical grey dashed lines highlight about when the NDDG changed diagnostic criteria. The GDM rise occurs in the context of changing criteria, with little impact on the PDM trend.

Within race/ethnic groups, admission rates rose slightly for PDM and sharply for GDM. By period end, $2.2 \%$ of Black women admitted while pregnant had PDM (Figure 2.A.1), the highest rate for that condition. On the other hand, the percent of admissions for pregnant Black women with GDM rose to 6.6\%, with Asian/PI women having the highest rate at $14 \%$ (Figure 2.B.1). For women age 35-44, PDM dropped to $1.4 \%$ then rose to $2.3 \%$ (Figure 2.A.2), while the rate for women age 35-44 with GDM rose 7 -fold from $2 \%$ to $14 \%$ (Figure 2.B.2).

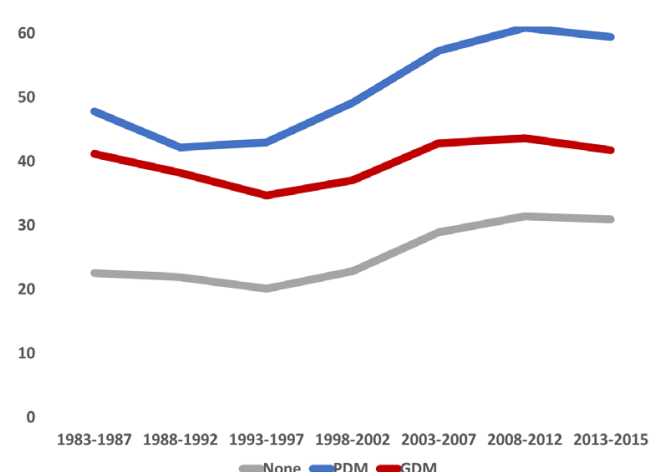

$\mathbf{A}$

Figure 3. Complications per 100 deliveries of a live infant (\%) by diabetes status 1983-2015 A. Hypertension in pregnancy (DXCL183)

B. Cesarean-section delivery (PXCL134)

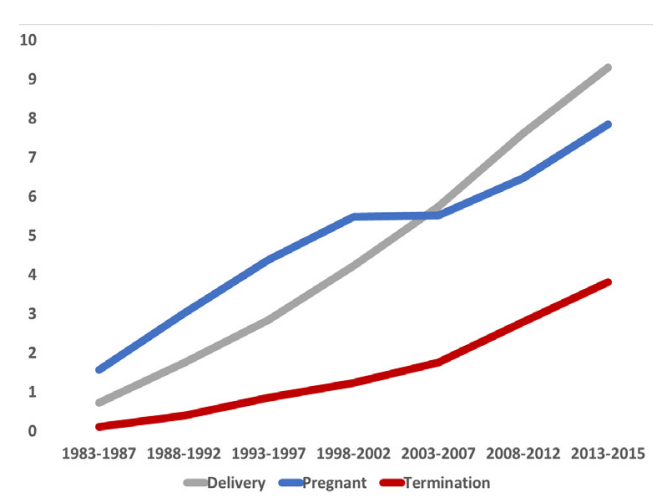

A

B

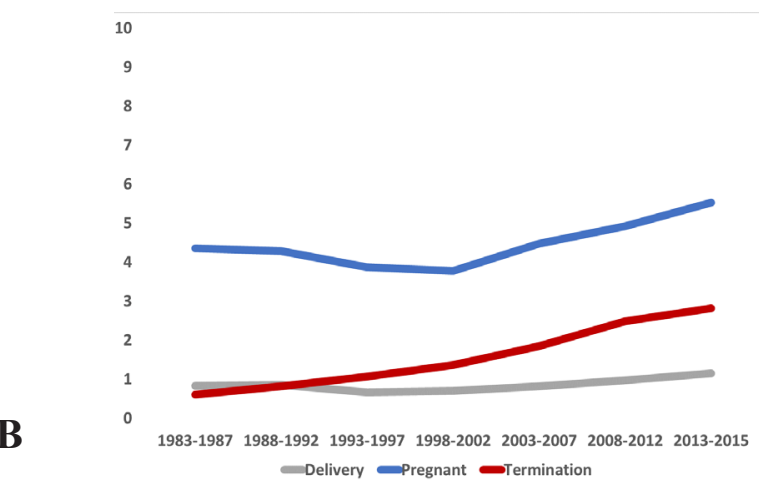

Figure 4. Pregnancy admission outcomes (\%) 1983-2015 by diabetes status

A. Pre-existing diabetes mellitus (PDM)

B. Gestational diabetes mellitus (GDM)

Overall, 2,203,926 admissions (11.6\%) did not result in live birth but ended by return home still pregnant $(7.7 \%)$ or after pregnancy termination (in-hospital abortion, miscarriage, or infant death at delivery) (3.9\%). Figure 4 shows outcome trends by diabetes status. Outcomes examined include whether the admission resulted in live delivery, whether the woman was discharged still pregnant, or whether the admission ended with pregnancy termination.

Figure 4A shows that among admissions resulting in delivery, about $1 \%$ had PDM and the rate stayed relatively flat. Among admissions ending with the woman still pregnant, the PDM rate rose from $4.4 \%$ to $5.5 \%$. However, among women whose admission ended with termination, the PDM rate rose from $0.6 \%$ to $2.8 \%$. Additionally, over the period, compared to women without diabetes, women with PDM were more likely to die ( $\mathrm{OR}=3.43$, CI 2.81- 4.19).

Figure $4 \mathrm{~B}$ shows sharp GDM increases from rates of $1 \%$ or less at start-of-period across all pregnancy admission outcomes. By period end, GDM was a factor in 9.3\% of admissions ending in delivery, $7.8 \%$ resulting in return home still pregnant, and 3.8\% resulting in pregnancy termination. Compared to women without diabetes, women with GDM were neither more nor less likely to die $(\mathrm{OR}=0.89$, CI 0.73- 1.09). 


\section{Conclusions}

Conversion from ICD-9-CM to ICD-10-CM in October, 2015 provided an opportunity to review hospital-based rates and trends for PDM and GDM. Today about 1 in 10 California women admitted to hospital while pregnant have one of these conditions. Women with GDM have increased risk for adverse events in the current and later pregnancies and are at increased risk of converting to PDM as they age $[6,18]$. Whether mothers have PDM or GDM, infants they conceive have increased risk of short- and long-term adverse outcomes [19,20]. With birth rates increasing among women older than 30 [21] and obesity increasing in the reproductive age population [22], current estimates are that $20-50 \%$ of women with GDM will have PDM within 10 years [4].

Several recent reports have focused on the relationship between rate changes and the timing of now seven position statements since 1979, released by the American Diabetes Association and/or the European Association for the Study of Diabetes $[3,10]$. Do reports of diabetes increasing in pregnant women reflect real underlying changes in population health or changes in professional attention to these conditions? Trends may be rising because of different diagnostic criteria, because of underlying changes in risk factors, or perhaps for both reasons. Regardless of why trends are rising, PDM and GDM pose significant risk to a growing proportion of pregnant women.

\section{Acknowledgements}

Author contributions: LR conceived the study, made tables and figures, and wrote the first manuscript draft. TC wrote all SAS macros. Both TC and LR wrote or co-wrote SAS programs and contributed to manuscript revisions.

Guarantors name: Linda Remy.

Financial support: This work was done in the public interest without funding.

Conflict of interest: The authors have no conflicts of interest.

Data access: This work was covered by the following protocols: IRB 10-05122, Reference 155262, Committee on Human Research, University of California, San Francisco; Project 13-02-1077, Committee for the Protection of Human Subjects, State of California Health and Human Services Agency; and Request 2140325-01, Office of Statewide Health Planning and Development, State of California Health and Human Services Agency. Protocol restrictions do not allow data sharing. However, researchers with the same files [13] and our programs (available on request) would be able to replicate our findings.

\section{References}

1. Expert Committee on the Diagnosis and Classification of Diabetes Mellitus. Report of the expert committee on the diagnosis and classification of diabetes mellitus. Diabetes Care. 2003;26 Suppl 1:S5-S20.

2. National Diabetes Statistics Report, 2014. Last access 02-Sep2019 at: https://www.cdc.gov/diabetes/pdfs/data/2014-reportestimates-of-diabetes-and-its-burden-in-the-united-states.pdf

3. Classification and diagnosis of diabetes mellitus and other categories of glucose intolerance. National Diabetes Data Group. Diabetes. 1979;28(12):1039-1057.

4. Casagrande SS, Linder B, Cowie CC. Prevalence of gestational diabetes and subsequent Type 2 diabetes among U.S. women. Diabetes Res Clin Pract. 2018;141:200-208.

5. Bellamy L, Casas JP, Hingorani AD, Williams D. Type 2 diabetes mellitus after gestational diabetes: a systematic review and meta-analysis. Lancet. 2009;373(9677):1773-1779.

6. Kim C, Ferrara A. Gestational Diabetes During and After Pregnancy. London, UK: Springer, 2010.

7. Bryson CL, Ioannou GN, Rulyak SJ, Critchlow C. Association between gestational diabetes and pregnancy-induced hypertension. Am J Epidemiol. 2003;158(12):1148-1153.

8. Jain AP, Gavard JA, Mostello DJ, Rice JJ, Catanzaro RB, Hopkins SA. Characteristics of Recurrent Large-for-Gestational-Age Infants in Obese Women. Am J Perinatol. 2016;33(9):918-924.

9. Sobngwi E, Boudou P, Mauvais-Jarvis F, et al. Effect of a diabetic environment in utero on predisposition to type 2 diabetes. Lancet. 2003;361(9372):1861-1865.

10. Xiang AH. Association of Maternal Diabetes With Autism in Offspring. JAMA. 2017;317(5):537-538.

11. Lavery JA, Friedman AM, Keyes KM, Wright JD, Ananth CV. Gestational diabetes in the United States: temporal changes in prevalence rates between 1979 and 2010. BJOG. 2017;124(5):804813.

12. Remy L, Oliva G, Clay T (2008) Maternal morbidity and outcomes including mortality, California 2001-2006. Family Health Outcomes Project, University of California San Francisco. Available at: https://fhop.ucsf.edu/fhop-publicationshospitalizations-trends-and-outcomes

13. Remy L, Byers V, Clay T (2017) Reproductive outcomes after non-occupational exposure to hexavalent chromium, Willits California, 1983-2014. Environ Health. 2017;16(1):18.

14. Office of Statewide Health Planning and Development, (OSHPD). Request Data. Last access 18 Feb 2020 https://oshpd. ca.gov/data-and-reports/request-data/

15. Elixhauser A, Steiner C, Palmer L. Clinical Classifications Software (CCS), 2014. US Agency for Healthcare Research and Quality. http://www.hcup-us.ahrq.gov/toolssoftware/ccs/ccs. jsp. Accessed 02-Sep-2019.

16. Fingar KR, Mabry-Hernandez I, Ngo-Metzger Q, Wolff T, Steiner CA. Elixhauser A. Delivery Hospitalizations Involving Preeclampsia and Eclampsia, 2005-2014. HCUP Statistical Brief \#222. April 2017. Agency for Healthcare Research and Quality, Rockville, MD. Last access 08-Sep-2019 at: www.hcup-us.ahrq. gov/reports/statbriefs/sb222-Preeclampsia-Eclampsia DeliveryTrends.pdf

17. Fethney J. Statistical and clinical significance, and how to use confidence intervals to help interpret both. Aust Crit Care. 2010;23(2):93-97.

18. Bryson CL, Ioannou GN, Rulyak SJ, Critchlow C. Association between gestational diabetes and pregnancy-induced hypertension. Am J Epidemiol. 2003;158(12):1148-1153.

19. Crane SS, Wojtowycz MA, Dye TD, Aubry RH, Artal R. Association between pre-pregnancy obesity and the risk of cesarean delivery. Obstet Gynecol. 1997;89(2):213-216.

20. Xiong X, Saunders LD, Wang FL, Demianczuk NN. Gestational diabetes mellitus: prevalence, risk factors, maternal and infant outcomes. Int J Gynaecol Obstet. 2001;75(3):221-228.

21. Martin JA, Hamilton BE, Osterman MJK, Driscoll AK, Drake P. Births: final data for 2016. National vital statistics reports: from the Centers for Disease Control and Prevention, National Center for Health Statistics, National Vital Statistics System. 2018;67(1):1-55

22. Flegal KM, Kruszon-Moran D, Carroll MD, Fryar CD, Ogden CL. Trends in Obesity Among Adults in the United States, 2005 to 2014. JAMA. 2016;315(21):2284-2291. 